\title{
COMPOSIÇÃO ICTIOFAUNÍSTICA DA ÁREA DE MANGUEZAL DA BAÍA DE GUARATUBA, PARANÁ, BRASIL ${ }^{1}$
}

\author{
Paulo de Tarso da Cunha Chaves ${ }^{2}$ \\ Marco Fábio Maia Corrêa ${ }^{3}$
}

\begin{abstract}
Fish FAUNAL COMPOSITION OF THE MANGROVE AREA OF THE GUARATUBA BAY, PARANÁ, BraziL. The Guaratuba Bay $\left(25^{\circ} 52^{\prime} \mathrm{S}, 48^{\circ} 39^{\prime} \mathrm{W}\right.$ ) is the second largest estuarine system of the State of Parana, Brazil. It extended inside of the continent for about $15 \mathrm{~km}$ and is surrounded by mangroves. Water depth can reach up to six meters. The fish faunal composition of this area was evaluated with a bottom trawl and, occasionally, casting and gill nets. One species of Chondrichthyes and 59 of the Actinopterygii ( 50 genera and 28 families) were reported from the Bay. The results were not significantly different from those of the Paranagua Bay, except by the lower number of species. These differences appear related to distinct collection efforts and the extension of surveyed areas. No species can be considered endemic to the region. Tipically freshwater species were not collected.

KEY WORDS. Fish faunal composition, mangrove, Guaratuba Bay, Paraná, Brazil
\end{abstract}

A Baía de Guaratuba (2552'S, $\left.48^{\circ} 39^{\prime} \mathrm{W}\right)$ é o segundo maior sistema estuarino do litoral do estado do Paraná, sul do Brasil. Comunica-se com o Oceano Atlântico por uma abertura de aproximadamente 500 metros, e prolonga-se para dentro do continente por cerca de 15 quilômetros. Sua vegetação é própria de regiões de mangue, e a profundidade da lâmina d'água, em alguns poços, alcança 6 metros. A salinidade da água de fundo apresenta variação sazonal, atingindo valores mais altos no inverno e mais baixos no verão (respectivamente 37 e $3 \%$ ), enquanto a temperatura, por sua vez, pode ultrapassar $28^{\circ} \mathrm{C}$ e baixar a pelo menos $15^{\circ} \mathrm{C}$, conforme a estação (CHAVES \& VENDEL 1997b).

Artigos inventariando a fauna ictíica da Baía de Guaratuba são ainda incipientes. Afora uma listagem elaborada por JAKOBI \& SoUZA (1968), numa abordagem sócio-pesqueira abrangente para todo o litoral do Paraná, os demais trabalhos têm caráter essencialmente autoecológico, como os de CHAvES $(1994,1995)$ e Chaves \& VEndel (1996, 1997a,b). Assim, considerando a importância da Baía para as pescas esportiva e artesanal, e objetivando subsidiar ações de investigação e legislação pesqueiras, apresentam-se a seguir os resultados de um levantamento sobre as espécies de peixes ocorrentes na área de manguezal e adjacências.

1) Contribuição número 994 do Departamento de Zoologia, Universidade Federal do Paraná. Realizado com auxílios do CNPq e Funpar.

2) Departamento de Zoologia, Universidade Federal do Paraná. Caixa Postal 19020, 81531-990 Curitiba, Paraná, Brasil. Bolsista do CNPq.

E-mail: poissons@cce.ufpr.br

3) Centro de Estudos do Mar, Universidade Federal do Paraná.

E-mail:marco@cce.ufpr.br

Revta bras. Zool. 15 (1): 195 - 202, 1998 


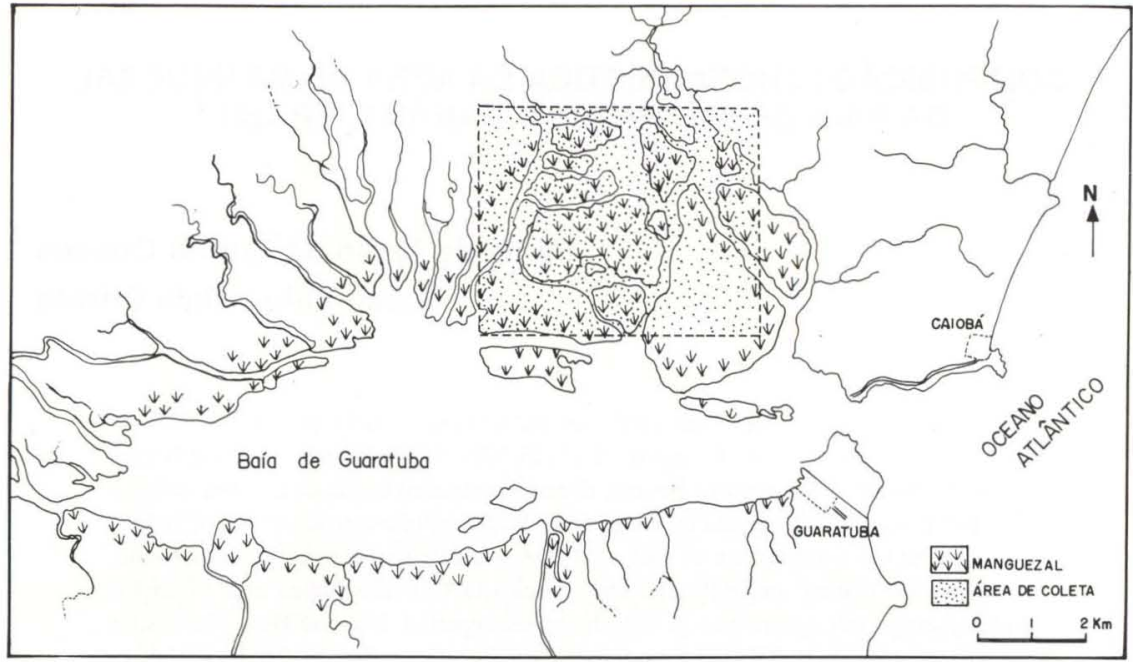

Fig. 1. Indicação da área de estudo (retângulo pontilhado), na Baia de Guaratuba, litoral do Estado do Paraná (2552'S;48³9'W). No detalhe, o círculo aponta a localização do sistema na costa brasileira.

\section{MATERIAL E MÉTODOS}

As coletas foram realizadas mensalmente, entre julho de 1993 e dezembro de 1996 (exceto maio/95), na área de mangue da Baía de Guaratuba (Fig. 1). Efetuaram-se arrastos de fundo com portas, utilizando rede de $1,5 \mathrm{~m}$ de abertura de boca e malha de $20 \mathrm{~mm}$ de abertura no ensacador (nós opostos). Em três oportunidades empregaram-se, também, tarrafa e rede de emalhe com diferentes malhagens. Imediatamente depois da captura, o material foi resfriado e transportado para Curitiba, distante $100 \mathrm{~km}$ do local de coleta. Em laboratório, após pesagem e biometria (comprimento total), indivíduos representativos de cada espécie foram fixados em solução de formalina a $10 \%$ e, passadas algumas semanas, transferidos para lotes em álcool a $70^{\circ} \mathrm{G}$.L.. Os exemplares integrantes de cada lote provêm, assim, de uma mesma expedição de coleta. A identificação do material seguiu trabalhos de referência para peixes marinhos da costa atlântica sul-americana, como FigueIREDO (1977), FisHeR (1978), FigueIREDO \& MENEZES (1978, 1980), MENEZES \& Figueiredo (1980, 1985), CORRÊA (1987) e CERVIGÓN et al. (1992).

Na relação a seguir apresentada, as espécies encontram-se listadas conforme sequiência de grandes grupos e de famílias adotada pelas publicações acima [exceto Fisher (1978) e CERVIGÓN et al. (1992)]. Dentro das famílias, as espécies estão dispostas em ordem alfabética. Para cada um dos exemplares examinados, são citados seu código de localização na coleção e o comprimento total do menor e do maior exemplar depositados. O código de localização é alfanumérico, compreendendo, na seqüência, cinco letras (MHNCI) e quatro algarismos arábicos. Adiciona-se ao final o nome comum da espécie na região. $\mathrm{O}$ material analisado encontra-se depositado no Museu de História Natural Capão da Imbuia, em Curitiba, Brasil. 


\section{RESULTADOS}

Foram registradas na região de estudo uma espécie da classe Chondrichthyes e 59 de Actinopterygii, estas distribuídas em 50 gêneros e 28 famílias.

\section{Chondrichthyes}

Rajiformes

Rhinobatidae

Rhinobatos percellens (Walbaum, 1792). MHNCI 8315 e 8316. 274$440 \mathrm{~mm}$. Cação-viola, raia-viola.

\section{Actinopterygii}

Anguilliformes

Muraenidae

Gymnothorax ocellatus Agassiz, 1834. MHNCI 8189. 440mm. Moréia. Clupeiformes

Clupeidae

Harengula clupeola (Cuvier, 1829). MHNCI 8190 a $8193.137-142 \mathrm{~mm}$.

Sardinha-cascuda.

Opisthonema oglinum (Lesueur, 1817). MHNCI 8194. 136mm. Sardinhabandeira.

Pellona harroweri (Fowler, 1917). MHNCI 8195. 140mm. Sardinha. Engraulididae (Engraulidae por alguns autores)

Cetengraulis edentulus (Cuvier, 1829). MHNCI 8196 a 8198. 128-148mm. Manjuba.

Lycengraulis grossidens (Cuvier, 1829). MHNCI 8199 a 8201. 158-241 mm. Manjubão.

Siluriformes

Ariidae

Cathorops spixii (Agassiz, 1829). MHNCI 8202 a 8205. 120-186mm. Bagre. Genidens genidens (Valenciennes, 1839). MHNCI 8206 e 8207. 193$208 \mathrm{~mm}$. Bagre.

Netuma barba (Lacepède, 1803). MHNCI 8208 a $8216.110-142 \mathrm{~mm}$. Bagre.

Sciadeichthys luniscutis (Valenciennes, 1840). MHNCI 8217. $355 \mathrm{~mm}$. Bagre.

Aulopiformes

Synodontidae

Synodus foetens (Linnaeus, 1766). MHNCI 8218. 265mm. Peixe-lagarto. Gasterosteiformes

Syngnathidae

Hippocampus reidi Ginsburg, 1933. MHNCI 8219 e $8220.65-80 \mathrm{~mm}$. Cavalo-marinho. 
Scorpaeniformes

Triglidae

Prionotus punctatus (Bloch, 1797). MHNCI 8221 e 8222. 102-156mm. Cabrinha.

Dactylopteriformes

Dactylopteridae

Dactylopterus volitans (Linnaeus, 1758). MHNCI 8223 e 8224. 170$224 \mathrm{~mm}$. Voador.

Perciformes

Centropomidae

Centropomus parallelus Poey, 1860. MHNCI 8225. 135mm. Robalo.

C. undecimalis (Bloch, 1792). MHNCI 8226 e 8227. 228-293mm. Robalo. Serranidae

Diplectrum radiale (Quoy \& Gaimard, 1824). MHNCI 8228.99mm. Michole.

Epinephelus itajara (Lichtenstein, 1822). MHNCI 8229.300mm. Mero.

E. niveatus (Valenciennes, 1828). MHNCI 8230. 148mm. Badejo.

Grammistidae

Rypticus randalli Courtenay, 1967. MHNCI 8231 e 8232.95-99mm. Peixesabão.

Pomatomidae

Pomatomus saltator (Linnaeus, 1766). MHNCI 8233 a 8235. 275-310mm. Anchova.

Carangidae

Caranx hippos (Linnaeus, 1766). MHNCI 8236. 195mm. Xerelete.

C. latus Agassiz, 1831. MHNCI 8237. 190mm. Xerelete.

Chloroscombrus chrysurus (Linnaeus, 1766). MHNCI 8238. 135mm. Palombeta.

Selene vomer (Linnaeus, 1758). MHNCI 8239 a 8243. 70-183mm. Peixegalo.

Trachinotus carolinus (Linnaeus, 1766). MHNCI 8244. 257mm. Pampo.

Gerreidae

Diapterus rhombeus (Cuvier, 1829). MHNCI 8245 a $8247.113-167 \mathrm{~mm}$. Caratinga.

Eucinostomus argenteus Baird \& Girard, 1854. MHNCI 8248. 130mm. Escrivão.

E. gula (Cuvier, 1830). MHNCI 8249 e 8250 . 116-121mm. Escrivão.

E. melanopterus (Bleeker, 1863). MHNCI 8251 e 8252. 110-180mm. Escrivão.

Eugerres brasilianus (Cuvier, 1830). MHNCI 8253. 180mm. Caratinga.

Haemulidae (Pomadasyidae incluída)

Genyatremus luteus (Bloch, 1795). MHNCI 8254 e $8255.167-288 \mathrm{~mm}$. 


\section{Saguá.}

Orthopristis ruber (Cuvier, 1830). MHNCI 8256 a 8259. 99-155mm. Corcoroca.

Pomadasys corvinaeformis (Steindachner, 1868). MHNCI 8260 a 8262. 90-175mm. Corcoroca.

Sparidae

Archosargus rhomboidalis (Linnaeus, 1758). MHNCI 8263. 238mm. SarSciaenidae go-de-dente.

Bairdiella ronchus (Cuvier, 1830). MHNCI 8264 e 8265. 155-156mm. Oveva.

Cynoscion acoupa (Lacepède, 1802). MHNCI 8266. 285mm. Pescada.

C. leiarchus (Cuvier, 1830). MHNCI 8267. 166mm. Pescada-branca.

Isopisthus parvipinnis (Cuvier 1830). MHNCI 8268. 195mm. Tortinha, pescadinha.

Menticirrhus americanus (Linnaeus, 1758). MHNCI 8269 a 8273. 55$131 \mathrm{~mm}$. Betara.

M. littoralis (Holbrook, 1860). MHNCI 8314. 110mm. Betara.

Micropogonias furnieri (Desmarest, 1823). MHNCI 8274. 239mm. Corvina.

Paralonchurus brasiliensis (Steindachner, 1875). MHNCI 8275. 195mm. Maria-Luísa.

Stellifer rastrifer (Jordan, 1889). MHNCI 8276 e 8277. 144-156mm. Cangulo.

Ephippidae

Chaetodipterus faber (Broussonet, 1782). MHNCI 8278 e 8279. 105$106 \mathrm{~mm}$. Paru.

Mugilidae

M. curema Valenciennes, 1836. MHNCI 8280 a 8282. 180-298mm. Tainha, parati.

M. gaimardianus Desmarest, 1831. MHNCI 8283.162mm. Parati.

Gobiidae

Bathygobius soporator (Valenciennes, 1837). MHNCI 8284. 110mm. Amborê.

Trichiuridae

Trichiurus lepturus Linnaeus, 1758. MHNCI 8285. 800mm. Peixe-espada. Pleuronectiformes

Paralichthyidae (Bothidae para alguns Autores)

Citharichthys arenaceus Evermann \& Marsh, 1900. MHNCI 8286 a 8291. 100-170mm. Linguado.

C. spilopterus Gunther, 1862. MHNCI 8292. 105mm. Linguado. Bothidae

Etropus crossotus Jordan \& Gilbert, 1882. MHNCI 8293 a 8295. 108- 
$123 \mathrm{~mm}$. Linguado.

Cynoglossidae

Symphurus plagusia (Bloch \& Schneider, 1801). MHNCI 8296 e 8297. 135-163mm. Língua-de-mulata.

Achiridae (Soleidae para alguns Autores)

Achirus lineatus (Linnaeus, 1758). MHNCI 8298 e 8302. 75-109mm. Linguado.

Trinectes paulistanus (Ribeiro, 1915). MHNCI 8303. 180mm. Linguado. Tetraodontiformes

Balistidae

Stephanolepis hispidus (Linnaeus, 1766). MHNCI 8304 e 8305. 63-80mm. Peixe-porco.

Diodontidae

Chilomycterus spinosus (Linnaeus, 1758). MHNCI 8306. 70mm. Baiacude-espinho.

Tetraodontidae

Lagocephalus laevigatus (Linnaeus, 1766). MHNCI 8307 a 8311.61 a $89 \mathrm{~mm}$. Baiacu-arara.

Sphoeroides testudineus (Linnaeus, 1758). MHNCI 8312 e 9313. 166$178 \mathrm{~mm}$. Baiacu-pintado.

\section{DISCUSSÃO}

Em termos numéricos, a quantidade de espécies registradas na área estuarina da Baía de Guaratuba acompanha a tendência geral descrita por VIEIRA \& MUSICK (1993), para esta latitude do Atlântico Ocidental e tais características do processo de amostragem. A relação entre número de espécies e número de famílias $(2,1)$, que segundo WHITFIELD (1994) fornece uma idéia do grau de diversificação dentro das famílias, é inferior ao observado nos manguezais de Guadeloupe $(2,31)$ por LoUIS (1983), mas está dentro do intervalo registrado por DIOUF (1996) nos estuários do oeste da África. Em relação ao Complexo Estuarino Baía de Paranaguá, 30 quilômetros ao norte (M.F.M. Corrêa comunicação pessoal), o número de espécies é reduzido em cerca de $60 \%$ das citações, fato que pode ser explicado em parte pelo menor esforço de captura que em Guaratuba foi dedicado às espécies pelágicas, e em parte por ali a área de estudo ter sido menor que a pesquisada em Paranaguá. Além disso, face às suas características de manguezal, a região estudada em Guaratuba apresenta valores de salinidade normalmente elevados, o que talvez explique a ausência de espécies associadas à água doce (ex.: Cichlidae e Cyprinodontiformes), localizadas por M.F.M. Corrêa (op. cit.) no outro sistema.

Com relação à natureza dos grupos e espécies registrados, os ocorrentes em Guaratuba são aqueles tradicionalmente conhecidos para a costa Sul-Sudeste do Brasil, ainda que diferenças pontuais possam ser detectadas. Três das famílias com maior representatividade em Guaratuba - Ariidae, Gerreidae e Sciaenidae - são também comuns em Paranaguá, e estão entre as sete citadas por VIEIRA \& MUSICK 
(1994) como sendo, nos estuários do Atlântico Ocidental entre $35^{\circ} \mathrm{S}$ e $37^{\circ} \mathrm{N}$, responsáveis por $80 \%$ do total de peixes coletados em arrasto de fundo. Entretanto, os escrivães Eucinostomus argenteus e E. melanopterus, o roncador Bairdiella ronchus $\mathrm{e}$ o linguado Citharichthys arenaceus são muito comuns no manguezal em Guaratuba, mas não foram encontrados por CHAO et al. (1982) na região estuarina da Lagoa dos Patos $\left(32^{\circ} \mathrm{S}\right)$, nem por ANDREATA et al. $(1989,1992)$ nas Lagunas de Jacarepaguá e Marapendi $\left(22^{\circ} \mathrm{S}\right)$. Inversamente, espécies de Atherinidae, comuns na Lagoa dos Patos e nas Lagunas de Jacarepaguá e Marapendi, bem como de Gadiformes na primeira, não foram localizadas no manguezal de Guaratuba. Deve ser apontado, porém, que observações pessoais atestam a ocorrência de peixes-rei em áreas abertas da Baía, notadamente na pesca com anzol, indicando que pode haver influência do aparelho de captura sobre o registro ou não deste grupo dentro do mangue. Quanto às abróteas, observações pessoais indicam a ocorrência na área de praia externa à Baía, bem como no interior da Baía de Paranaguá. Portanto, sua presença ao menos eventual no mangue de Guaratuba não pode ser descartada.

Nenhuma das espécies registradas em Guaratuba é considerada endêmica deste sistema. A explotação econômica de algumas delas pode ser viável, dependendo de estudos particulares sobre seu ciclo de vida na região. O tamanho que os indivíduos atingem na Baía de Guaratuba é em várias dessas espécies superior ao registrado neste trabalho, que menciona apenas o comprimento de exemplares tombados em coleção. Para informações biológicas já disponíveis sobre Genidens genidens, Bairdiella ronchus, Stellifer rastrifer, Citharichthys arenaceus e C. spilopterus, podem ser consultados os trabalhos de CHAvES $(1994,1995)$ e CHAVES \& VENDEL (1996, 1997a,b).

AGRADECIMENTOS. Pela colaboração prestada nas coletas e no processamento do material, os autores agradecem aos acadêmicos Ana Lúcia Vendel, Adriana Portes Santos, Adriana Serenato, Gislaine Otto e Geraldo Simião, da Universidade Federal do Paraná. Ainda, a dois Revisores anônimos.

\section{REFERÊNCIAS BIBLIOGRÁFICAS}

ANDREATA, J.V.; A.M. SAAD \& L.R.R. BARBIERI. 1989. Associação e distribuição das espécies de peixes na Laguna de Marapendi, Rio de Janeiro, no período de março de 1985 a fevereiro de 1987. Mem. Inst. Oswaldo Cruz, Rio de Janeiro, 84 (Supl. IV): 45-51.

Andreata, J.V.; L.A. Moraes; C.L. SoAres \& A.G. MarCA. 1992. Associações, similaridade e abundância relativa dos peixes da Laguna de Jacarepaguá, Rio de Janeiro, Brasil. Bol. Mus. Nac., N.S. Zool., Rio de Janeiro, (355): 1-25.

Cervigón, F.; R. Cipriani; W. Fischer; L. Garibaldi; M. Hendrickx; A.J. Lemus; R. MÁrquez; J.M. Poutiers; G. Robaina \& B. Rodriguez. 1992. Guia de Campo de las Especies Comerciales Marinas y de Águas Salobres de la Costa Septentrional de Sur America. FAO, Roma, 513p.

Chao, L.N.; L.E. PereirA; J.P. VieIRA; M.A. BEMVENUTI \& L.P.R. CUNHA. 1982. Relação preliminar dos peixes estuarinos e marinhos da Lagoa dos Patos e região costeira adjacente, Rio Grande do Sul, Brasil. Atlântica, Rio Grande, 5: 67-75. Chaves, P.T.C. 1994. A incubação de ovos e larvas em Genidens genidens 
(Valenciennes) (Siluriformes, Ariidae) da Baía de Guaratuba, Paraná, Brasil. Revta bras. Zool. 11 (4): 641-648.

. 1995. Atividade reprodutiva de Bairdiella ronchus (Cuvier) (Pisces,

Sciaenidae) na Baía de Guaratuba, Paraná, Brasil. Revta bras. Zool. 12 (4): 759-766.

Chaves, P.T.C. \& A.L. Vendel. 1996. Aspectos da alimentação de Genidens genidens (Valenciennes) (Siluriformes, Ariidae) na Baía de Guaratuba, Paraná. Revta bras. Zool. 13 (3): 669-675.

1997a. Indicadores reprodutivos das espécies de Citharichthys Bleeker

(Teleostei, Pleuronectiformes) na Baía de Guaratuba, Paraná, Brasil. Revta bras. Zool. 14 (1): 73-79.

. 1997b. Reprodução de Stellifer rastrifer (Jordan) (Teleostei, Sciaenidae) na Baía de Guaratuba, Paraná, Brasil. Revta bras. Zool. 14 (1): 81-89.

DiOUF, P.S. 1996. Les peuplements de poissons des milieux estuariens de

l'Afrique de l'ouest: l'exemple de l'estuaire hyperalin du Sine-Saloum.

Thèse, Université Montpellier II, França, 178p.

Figueiredo, J.L. 1977. Manual de Peixes Marinhos do Sudeste do Brasil. I.

Introdução. Cações, Raias e Quimeras. São Paulo, Museu de Zoologia, Universidade de São Paulo, 105p.

Figueiredo, J.L. \& N.A. Menezes. 1978. Manual de Peixes Marinhos do Sudeste do Brasil. II. Teleostei (1). São Paulo, Museu de Zoologia, Universidade de São Paulo, 110p.

- 1980. Manual de Peixes Marinhos do Sudeste do Brasil. III. Teleostei

(2). São Paulo, Museu de Zoologia, Universidade de São Paulo, 90p.

FISHER, W. 1978. FAO Species Identification Sheets for Fishery Purposes.

Western Central Atlantic (Fishing Area 31). Roma, FAO, V: 1-7.

JAKOBI, H. \& E.A. SouZA. 1968. Contribuição ao conhecimento da pesca no Paraná.

Boletim da Universidade Federal do Paraná - Zoologia, Curitiba, 2 (14): 329-358.

Louis, M. 1983. Biologie, Ecologie et Dynamique des Populations de Poissons dans les Mangroves de Guadeloupe (Antilles Françaises). Thèse de Doctorat d'Etat, Univ. Sci. Tech. Languedoc, Montpellier II, França. 275p.

Menezes, N.A. \& N.A. Figueiredo. 1980. Manual de Peixes Marinhos do Sudeste do Brasil. IV. Teleostei (3). São Paulo, Museu de Zoologia da Universidade de São Paulo, 96p.

1985. Manual de Peixes Marinhos do Sudeste do Brasil. IV. Teleostei

(4). São Paulo, Museu de Zoologia da Universidade de São Paulo, 105p.

VIEIRA, J.P. \& J.A. MUSICK. 1993. Latitudinal patterns in diversity of fishes in warm-temperate and tropical estuarine waters of the western Atlantic. Atlântica, Rio Grande, 15: 115-133.

. 1994. Fish faunal composition in warm-temperate and tropical estuaries of western Atlantic. Atlântica, Rio Grande, 16: 31-53.

WHITFIELD, A.K. 1994. A review of ichtyofaunal biodiversity in Southern African estuarine systems. In: G. TEUGELS; J.F. GUEGAN \& J.J. AlbareT (Eds). Diversité biologique des poissons d'eaux douces et saumâtres d'Afrique. Ann. Mus. R. Afr. Centr., Zool. 275: 149-163.

Recebido em 27.I.1997; aceito em 13.IV.1998. 